\title{
Validation of a clinical and genetic model for predicting severe COVID-19
}

3 Gillian S. Dite ${ }^{1 *}$ (ORCID: 0000-0002-2448-2548)

4 Nicholas M. Murphy ${ }^{1}$ (ORCID: 0000-0001-7881-1952)

$5 \quad$ Erika Spaeth ${ }^{2}$ (ORCID: 0000-0002-5397-3493)

6 Richard Allman ${ }^{1}$ (ORCID: 0000-0002-8635-5237)

$7 \quad$ Lifelines Corona Research Initiative ${ }^{3}$

9 1. Genetic Technologies Limited, Fitzroy, Victoria, Australia

10 2. Phenogen Sciences Inc, Charlotte, North Carolina, United States of America

11 3. Members of the Lifelines Corona Research Initiative are as follows: 
d. Department of Genetics, University of Groningen, University Medical Center

e. Center of Development and Innovation, University of Groningen, University

$31 *$ Corresponding author. Genetic Technologies Limited, 60-66 Hanover St, Fitzroy VIC

32 3065, Australia.

33 Email: gillian.dite@gtglabs.com

35 Word count: 2495

37 Key words: severe COVID-19, risk prediction, validation, risk factors, single-nucleotide

38 polymorphism 


\section{Summary}

40 Using nested case-control data from the Lifelines COVID-19 cohort, we undertook a

41 validation study of a clinical and genetic model to predict the risk of severe COVID-19 in

42 people with confirmed COVID-19 and in people with confirmed or self-reported COVID-19.

43 The model performed well in terms of discrimination of cases and controls for all ages (area

44 under the receiver operating characteristic curve $[\mathrm{AUC}]=0.680$ for confirmed COVID-19

45 and $\mathrm{AUC}=0.689$ for confirmed and self-reported COVID-19) and in the age group in which

46 the model was developed (50 years and older; AUC $=0.658$ for confirmed COVID-19 and

$47 \mathrm{AUC}=0.651$ for confirmed and self-reported COVID-19). There was no evidence of over- or

48 under-dispersion of risk scores but there was evidence of overall over-estimation of risk in all

49 analyses (all $P<0.0001$ ). In the light of large numbers of people worldwide remaining

50 unvaccinated and continuing uncertainty regarding vaccine efficacy over time and against

51 variants of concern, identification of people at high risk of severe COVID-19 may encourage

52 the uptake of vaccinations (including boosters) and the use of non-pharmaceutical inventions. 


\section{Text}

Severe coronavirus disease 2019 (COVID-19) disproportionately affects older adults,

55 but can occur in people of all ages, especially those with comorbidities [1]. An abundance of

56 research has identified clinical and genetic risk factors that are associated with developing

57 severe disease if infected with severe acute respiratory syndrome-coronavirus-2 (SARS-CoV-

58 2) $[1,2]$. In clinical practice, information on these risk factors can be useful when combined

59 in a risk prediction model that provides a single estimate of absolute risk that enables health

60 care providers to effectively communicate with their patients about risk.

61 We previously described a clinical and genetic model for predicting severe COVID-

6219 that was developed and validated using data from the UK Biobank [3]. We now report the

63 results of a validation of the model using a case-control analysis of an external dataset from

64 the Netherlands [4]. While the risk prediction model was developed in people aged 50 years

65 and older, here we assess its performance in people aged 24 years and older.

66 We used data from participants in the Lifelines COVID-19 cohort [4] who were

67 recruited from the Lifelines and Lifelines NEXT cohorts [5, 6]. Lifelines is a multi-

68 disciplinary prospective population-based cohort study examining, in a unique three-

69 generation design, the health and health-related behaviours of 167,729 people living in the

70 north of the Netherlands. Lifelines employs a broad range of investigative procedures in

72 that contribute to the health and disease of the general population, with a special focus on

73 multi-morbidity and complex genetics. 
78 the regular online COVID-19 questionnaires via an emailed link during the first eight weeks

79 of data collection [4].

80 The questionnaire response dates corresponded to the period from around one month

81 after the beginning of the first wave of the COVID-19 pandemic in the Netherlands through

82 to the peak of the fourth wave in May 2021. During this time, the original SARS-CoV-2 virus

83 accounted for over $95 \%$ of infections in the Netherlands until early January 2021, after which

84 the alpha variant became more prevalent and had accounted for over $95 \%$ of infections by the

85 end of March 2021 [7]. The presence of the delta variant was negligible during the period of

86 data collection for this study.

87 COVID-19 vaccinations became available in the Netherlands in mid-January 2021

88 and were initially offered to high-risk groups and then progressively to other groups (such as

89 care workers) and younger age groups until all adults became eligible in mid-June 2021 [8].

90 From questionnaire 18 (March 2021) onwards, participants were asked about their

91 vaccination status and we excluded questionnaires where (and after which) a participant

92 reported having had one or two doses of a vaccine.

93 At the beginning of data collection, when testing for SARS-CoV-2 infection was not

94 widely available in the Netherlands, Lifelines COVID-19 questionnaires 1-4 asked

95 participants whether a doctor had told them they had COVID-19 [4]. From questionnaire 5

96 (early May 2020) onwards, the questionnaires also asked about positive test results. From

97 these questions we identified a group of participants with confirmed COVID-19. In addition,

98 the questionnaires asked participants to self-report having had COVID-19. We used this

99 question with the previous questions to identify a broader group of participants who had

100 either confirmed or self-reported COVID-19.

101 Given the limited availability of testing early in the data collection period, the

102 confirmed COVID-19 group is likely to miss some participants who had COVID-19. 
103 Conversely, the broader group including participants with self-reported COVID-19 is likely

104 to have some false positives. The true number of participants who had COVID-19 will be

105 somewhere between the two. Therefore, we conducted two sets of analyses: (i) using

106 participants with confirmed COVID-19 and (ii) using participants with confirmed and self-

107 reported COVID-19.

108 As we did previously, we used hospitalization as a proxy for severe COVID-19 [3].

109 The Lifelines COVID-19 questionnaires specifically asked participants whether they had

110 been hospitalized for COVID-19. The questionnaires also asked about being given

111 supplemental oxygen, admission to an intensive care unit and being placed on a ventilator,

112 but there were too few positive responses to these questions to allow separate analysis.

113 The risk factors included in the calculation of the risk of severe COVID-19 are age;

114 sex; body mass index; a history of cerebrovascular disease, diabetes, haematological cancer,

115 non-haematological cancer, hypertension, kidney disease or respiratory disease (excluding

116 asthma); and the genotypes of seven single nucleotide polymorphisms (SNPs) -

117 rs112641600, rs10755709, rs118072448, rs7027911, rs71481792, rs112317747 and

118 rs2034831 [3]. The log odds of the risk of severe COVID-19 is the sum of the intercept and

119 the product of the value and beta coefficient for each of the risk factors listed in

120 Supplementary Table S1. The probability of severe COVID-19 is then the inverse logit of the

$121 \log$ odds $(x)$, that is, $\frac{1}{\left(1+e^{-x}\right)}$.

122 We used the age reported at the completion of the participant's first Lifelines COVID-

12319 questionnaire. The questionnaires asked about a history of cancer, cerebrovascular disease,

124 diabetes, hypertension, kidney disease and respiratory disease on three occasions. If any of

125 the participants' responses to the risk factor questions were missing for all answered

126 questionnaires, we used responses from their Lifelines baseline questionnaire. 
participants so we used the risk associated with having a non-haematological cancer for all

130 asthma, whereas this is excluded in the model calculations. Because we were not able to

131 distinguish respiratory disease solely due to asthma, we included all reports of respiratory

132 disease in the model calculations. Gender, ethnicity, weight and height were taken from the

133 Lifelines baseline questionnaire. If two weight or height measurements were available, we

134 used the most recent weight measurement and the mean of the height measurements. Two of

135 the SNPs in the risk model were not available on the Illumina CytoSNP-12v2 array used by

136 Lifelines [6]. Instead, we used highly correlated proxy SNPs (rs10905502 was the proxy for

$137 \mathrm{rs} 71481792\left[\mathrm{r}^{2}=0.75, \mathrm{D}^{\prime}=1.0\right]$ and $\mathrm{rs} 78654835$ was the proxy for $\mathrm{rs} 112317747\left[\mathrm{r}^{2}=1.0, \mathrm{D}^{\prime}\right.$

$138=1.0])$.

139 To extend the model to people aged less than 50 years, we estimated the risk

140 associated with younger age groups using data from the Centers for Disease Control and

141 Prevention [9] such that, compared with the 50-69 years baseline age group, people aged 18-

14229 years were at 0.27 times the risk, people aged 30-39 years were at 0.43 times the risk, and

143 people aged $40-49$ years were at 0.67 times the risk.

144 In each analysis - (i) using participants with confirmed COVID-19 and (ii) using

145 participants with both confirmed and self-reported COVID-19 - the cases were those who

146 reported having been hospitalized for COVID-19 and the controls were the remainder of the

147 group. We also did analyses restricting the dataset to those aged 50 years or older (the ages in

148 which the model was developed).

149 As we did previously [3], we assessed the association between quintile of risk score

150 and severe COVID-19 using logistic regression. We used the area under the receiver

151 operating characteristic curve (AUC) to assess discrimination. We used logistic regression of 
152 the log odds of the risk score to assess calibration in terms of the overall estimation of risk

153 (the intercept) and the dispersion of risk (the slope), and we drew calibration plots of deciles

154 of expected and observed cases of severe COVID-19. We used Stata MP version 13.1

155 (StataCorp LP, College Station, Texas, USA) for all analyses and all statistical tests were two 156 sided.

157 The Lifelines protocol has been approved by the Medical Ethical Committee of the

158 University Medical Center Groningen, The Netherlands, under Approval Number 2007/152.

159 All participants provided written informed consent to Lifelines before data collection began.

160 This research was conducted using Lifelines data under Project Number OV20-00101.

161 The data used in this study was made available to us by Lifelines and is not publicly

162 available. Researchers can apply to use the Lifelines data used in this study, and more

163 information about how to request Lifelines data and the conditions of use can be found on

164 their website (https://www.lifelines.nl/researcher/how-to-apply). Stata MP Version 13.1 code

165 for the analysis is available for non-commercial purposes from the corresponding author on

166 request.

167 Of the 26845 Lifelines COVID-19 cohort participants who had genotyping data

168 available and had completed at least one questionnaire, $3214(12.0 \%)$ completed one

169 questionnaire, $5742(21.4 \%)$ completed 2-5 questionnaires, 4194 (15.6\%) completed 6-10

170 questionnaires, $3568(13.3 \%)$ completed 11-15 questionnaires, 6106 (22.7\%) completed 16-

17120 questionnaires and 4021 (15.0\%) completed 21-23 questionnaires. We excluded 15933

172 questionnaires from 15040 participants where (and after which) the participant reported

173 being vaccinated.

174 In the final dataset, 55 participants were hospitalized for their COVID-19 infection

175 and were considered cases in this study. We used two control groups: the first comprised the

1761355 participants who had confirmed COVID-19; the second comprised both the first control 
medRxiv preprint doi: https://doi.org/10.1101/2022.01.14.22269270; this version posted January 15, 2022. The copyright holder for this preprint (which was not certified by peer review) is the author/funder, who has granted medRxiv a license to display the preprint in perpetuity.

It is made available under a CC-BY-NC-ND 4.0 International license .

177 group and the 2518 participants who self-reported having had COVID-19 (i.e. 3925

178 participants). In the cases, there were 28 (50.9\%) women and 27 (49.1\%) men; their mean

179 age was 57.6 years (standard deviation $[\mathrm{SD}]=10.3$ ) and the mean number of completed

180 questionnaires was $17.0(\mathrm{SD}=6.3)$. In the confirmed COVID-19 control group, there were

$181905(66.8 \%)$ women and $450(33.2 \%)$ men; their mean age was 53.0 years $(\mathrm{SD}=11.6)$ and

182 the mean number of completed questionnaires was $14.1(\mathrm{SD}=6.8)$. In the confirmed and

183 self-reported COVID-19 control group, there were 2414 (62.3\%) women and 1459 (37.7\%)

184 men; their mean age was 51.5 years $(\mathrm{SD}=11.8)$ and the mean number of completed

185 questionnaires was $12.2(\mathrm{SD}=7.2)$.

186 In the cases, the mean probability of severe COVID-19 was 0.225 (SD =0.019); in

187 the confirmed COVID-19 controls, the mean was $0.165(\mathrm{SD}=0.002)$; and in the confirmed

188 and self-reported COVID-19 controls, the mean was $0.165(\mathrm{SD}=0.001)$. The risk

189 distribution for the cases, both control groups and the whole Lifelines COVID-19 cohort are

190 show in in Supplementary Figure S1.

191 The top half of Table 1 shows the results of the analyses of the confirmed COVID-19

192 group and the confirmed and self-reported COVID-19 group for all ages. Overall, the results

193 were similar for the two groups. The odds ratios (OR) per quintile of risk (1.63 and 1.58,

194 respectively) were a little lower than the OR of 1.77 seen in the validation group in the risk

195 prediction model development paper [3]. Similarly, the AUCs ( 0.680 and 0.679 , respectively)

196 were a little lower than the AUC of 0.732 seen in the model development paper. In terms of

197 calibration, there was no evidence of under- or over-dispersion in either group $(\beta=0.92$ and

1980.90 , respectively), as in the original paper $(\beta=0.90)$. In both groups, the model

199 overestimated risk ( $\alpha=-1.78$ and -2.86 , respectively), whereas the validation group in the

200 model development paper did not $(\alpha=-0.08)$. 
medRxiv preprint doi: https://doi.org/10.1101/2022.01.14.22269270; this version posted January 15, 2022. The copyright holder for this preprint (which was not certified by peer review) is the author/funder, who has granted medRxiv a license to display the preprint in perpetuity.

It is made available under a CC-BY-NC-ND 4.0 International license .

201 The bottom half of Table 1 shows the analyses limited to participants aged 50 years

202 and older. Compared with the analysis of all ages, there was a reduction in the ORs per

203 quintile of risk for both the confirmed COVID-19 group and the confirmed and self-reported

204 COVID-19 group (1.54 and 1.47, respectively), and a reduction in the AUCs (0.658 and

205 0.651, respectively). The calibration slopes suggested over-dispersion of risk but were not

206 statistically significant. The overestimations of risk ( -1.84 and -2.87 , respectively) were

207 similar to those seen in the analysis of all ages.

208 The true number of people with COVID-19 is unknown but is likely to be somewhere

209 between the number who test positive for SARS-CoV-2 infection and the number who self-

210 report having had COVID-19. In this study we have addressed this uncertainty by conducting

211 two sets of analyses: the first in individuals with confirmed COVID-19, and the second with

212 individuals with confirmed and self-reported COVID-19. In terms of discrimination, the

213 AUC of the risk prediction model was almost identical in the two analyses and only slightly

214 lower than the AUC in validation group in the model development paper [3]. This and the

215 similarity in the association per quintile of risk (Table 1) provide confidence in the model's

216 application across adult populations. Risk of COVID-19 severity was overestimated in this

217 study, but in a clinical setting, overestimation of risk is preferred to an underestimation given

218 that the risk-reduction options of vaccination, masking and social distancing are benign in

219 nature. Our results were similar for the full dataset and when limiting analyses to people aged

22050 years and over.

221 It is possible that some severe cases of COVID-19 have not been ascertained in this

222 dataset. Death registry linkage identified 77 deaths in the broader Lifelines COVID-19 cohort

223 in people who did not have confirmed or self-reported COVID-19. While these deaths may

224 have been unrelated to COVID-19, some will represent people who became infected and 
medRxiv preprint doi: https://doi.org/10.1101/2022.01.14.22269270; this version posted January 15, 2022. The copyright holder for this preprint (which was not certified by peer review) is the author/funder, who has granted medRxiv a license to display the preprint in perpetuity.

It is made available under a CC-BY-NC-ND 4.0 International license .

225 were too unwell to complete a Lifelines COVID-19 questionnaire before they died. This

226 limitation may have attenuated some of the results seen in this study.

227 As the pandemic continues to evolve, there are two major issues that can affect the

228 utility of our risk model. First, we have to address the impact of viral variants on the

229 performance of the risk model. The model development paper [3] and the present study used

230 datasets in which the original and alpha SARS-CoV-2 variants were predominant. We have

231 not been able to assess our model in datasets with known delta or omicron SARS-CoV-2

232 variants. We hypothesize that the clinical and genetic risk factors have broad effects in terms

233 of risk of severe disease because the delta and omicron SARS-CoV-2 variants appear to

234 affect transmissibility rather than severity [10].

235 Second, our model does not incorporate the protection offered by vaccination. Thus in

236 vaccinated adults, the model will overestimate their risk of developing severe disease.

237 However, we know that vaccine immunity wanes over about six months through a steady

238 reduction in antibody levels leading to greater number of breakthrough infections among the

239 vaccinated [11]. The wide range of immunity across individuals makes it hard to predict the

240 impact of waning vaccination in terms of risk. Thus, we believe that the model can be used to

241 provide a baseline risk of developing severe disease, even in the context of vaccinated adults.

242 Herein, we have validated our model to predict risk of severe COVID-19 if infected

243 with SARS-CoV-2 in a dataset unrelated to the one in which the model was originally

244 developed and validated. Despite new SARS-CoV-2 variants of concern, the model may

245 complement current public health efforts in vaccine (and booster) uptake and may enable

246 healthcare providers to have more informed discussions with patients about their risk-

247 mitigation options and early treatment awareness, if ever infected. 


\section{References}

249 1. Gao YD, et al. (2021) Risk factors for severe and critically ill COVID-19 patients: A

$250 \quad$ review. Allergy; 76: 428-455.

251 2. Velavan TP, et al. (2021) Host genetic factors determining COVID-19 susceptibility 252 and severity. EBioMedicine; 72: 103629.

253 3. Dite GS, Murphy NM, Allman R. (2021) Development and validation of a clinical 254 and genetic model for predicting risk of severe COVID-19. Epidemiology and

$255 \quad$ Infection; 149: e162.

256 4. Mc Intyre K, et al. (2021) Lifelines COVID-19 cohort: investigating COVID-19

257 infection and its health and societal impacts in a Dutch population-based cohort. BMJ

$258 \quad$ Open; 11: e044474.

259 5. Warmink-Perdijk WDB, et al. (2020) Lifelines NEXT: a prospective birth cohort

260 adding the next generation to the three-generation Lifelines cohort study. European

$261 \quad$ Journal of Epidemiology; 35: 157-168.

262 6. Scholtens S, et al. (2015) Cohort Profile: LifeLines, a three-generation cohort study

263 and biobank. International Journal of Epidemiology; 44: 1172-1180.

2647 National Institute for Public Health and the Environment. Coronavirus variants.

265 Available at https://coronadashboard.government.nl/landelijk/varianten (Accessed 10

$266 \quad$ November 2021).

267 8. National Institute for Public Health and the Environment. COVID-19 vaccinations.

268 Available at https://coronadashboard.government.nl/landelijk/vaccinaties (Accessed 16

$269 \quad$ November 2021). 
medRxiv preprint doi: https://doi.org/10.1101/2022.01.14.22269270; this version posted January 15, 2022. The copyright holder for this preprint

(which was not certified by peer review) is the author/funder, who has granted medRxiv a license to display the preprint in perpetuity.

It is made available under a CC-BY-NC-ND 4.0 International license.

270 9. Centers for Disease Control and Prevention. COVIDView: a weekly surveillance

271 summary of U.S. COVID-19 activity. Available at

272 https://www.cdc.gov/coronavirus/2019-ncov/covid-data/pdf/covidview-08-07-2020.pdf

273 (Accessed 15 November 2021).

274 10. Lewis T. Omicron's effect won't be as mild as hoped. Scientific American; 23

275 December. Available at https://www.scientificamerican.com/article/omicrons-effect-

276 wont-be-as-mild-as-hoped1/ (Accessed 13 January 2021).

277 11. Cromer D, et al. (2021) Prospects for durable immune control of SARS-CoV-2 and

278 prevention of reinfection. Nature Reviews: Immunology; 21: 395-404. 


\section{Acknowledgements}

The authors wish to acknowledge the services of the Lifelines Cohort Study

281 (especially the data management team), the contributing research centres delivering data to

282 Lifelines and all the study participants.

\section{Financial support}

The Lifelines initiative has been made possible by subsidy from the Dutch Ministry of

285 Health, Welfare and Sport, the Dutch Ministry of Economic Affairs, the University Medical

286 Center Groningen, Groningen University and the Provinces in the North of the Netherlands

287 (Drenthe, Friesland, Groningen).

288 The analyses undertaken in this study were fully funded by Genetic Technologies

289 Limited.

\section{Conflict of interest}

GSD, NMM, and RA are employees of Genetic Technologies Limited. ES is an

292 employees of Phenogen Sciences Inc (a subsidiary of Genetic Technologies Limited).

293 Genetic Technologies Limited had no role in the conceptualization, design, data analysis,

294 decision to publish or preparation of the manuscript.

295 Aspects of this manuscript are covered by Provisional Patent Application

296 AU_2021900392 (pending), Methods of assessing risk of developing a severe response to

297 Coronavirus infection. GSD, NMM and RA are named inventors on the patent application,

298 which is assigned to Genetic Technologies Limited. 
300 Table 1. Validation analysis of model to predict risk of severe COVID-19 for participants of

301 all ages and for participants aged 50 years and older

\begin{tabular}{|c|c|c|c|}
\hline All ages & Estimate & $95 \% \mathrm{Cl}$ & $P$ value \\
\hline \multicolumn{4}{|l|}{ Association: OR per quintile of risk } \\
\hline Confirmed COVID-19 & 1.63 & $1.31,2.04$ & $<0.0001$ \\
\hline Confirmed and self-reported COVID-19 & 1.58 & $1.27,1.95$ & $<0.0001$ \\
\hline \multicolumn{4}{|l|}{ Discrimination: AUC } \\
\hline Confirmed COVID-19 & 0.680 & $0.608,0.752$ & \\
\hline Confirmed and self-reported COVID-19 & 0.679 & $0.607,0.751$ & \\
\hline \multicolumn{4}{|l|}{ Calibration slope: $\beta$} \\
\hline Confirmed COVID-19 & 0.92 & $0.54,1.30$ & $0.7^{*}$ \\
\hline Confirmed and self-reported COVID-19 & 0.90 & $0.55,1.25$ & $0.6^{*}$ \\
\hline \multicolumn{4}{|l|}{ Calibration intercept: $a$} \\
\hline Confirmed COVID-19 & -1.78 & $-2.38,-1.19$ & $<0.0001$ \\
\hline Confirmed and self-reported COVID-19 & -2.86 & $-3.41,-2.30$ & $<0.0001$ \\
\hline Aged 50 years and older & Estimate & $95 \% \mathrm{Cl}$ & $P$ value \\
\hline \multicolumn{4}{|l|}{ Association: OR per quintile of risk } \\
\hline Confirmed COVID-19 & 1.54 & $1.21,1.96$ & $<0.0001$ \\
\hline Confirmed and self-reported COVID-19 & 1.47 & $1.16,1.86$ & 0.001 \\
\hline \multicolumn{4}{|l|}{ Discrimination: AUC } \\
\hline Confirmed COVID-19 & 0.658 & $0.579,0.737$ & \\
\hline Confirmed and self-reported COVID-19 & 0.651 & $0.573,0.730$ & \\
\hline \multicolumn{4}{|l|}{ Calibration slope: $\beta$} \\
\hline Confirmed COVID-19 & 0.75 & $0.34,1.16$ & $0.2^{*}$ \\
\hline Confirmed and self-reported COVID-19 & 0.69 & $0.31,1.08$ & $0.1^{*}$ \\
\hline \multicolumn{4}{|l|}{ Calibration intercept: $a$} \\
\hline Confirmed COVID-19 & -1.85 & $-2.48,-1.21$ & $<0.0001$ \\
\hline Confirmed and self-reported COVID-19 & -2.87 & $-3.46,-2.28$ & $<0.0001$ \\
\hline
\end{tabular}

302 Note: AUC, area under the receiver operating characteristic curve; $\mathrm{Cl}$, confidence interval; $\mathrm{OR}$, odds 303 ratio. ${ }^{*} P$ value for the null hypothesis that the calibration slope $=1$. 


\section{Supplementary information}

305 Supplementary Table S1. Beta coefficients for calculation of risk of severe COVID-19

\begin{tabular}{|c|c|c|}
\hline Variable & Value & Beta coefficient \\
\hline Intercept & & -1.37 \\
\hline Age $18-29$ years & $0=$ no, $1=$ yes & -1.31 \\
\hline Age $30-39$ years & $0=$ no, $1=$ yes & -0.83 \\
\hline Age $40-49$ years & $0=$ no, $1=$ yes & -0.40 \\
\hline Age $50-69$ years & $0=$ no, $1=$ yes & 0.00 \\
\hline Age $70-74$ years & $0=$ no, $1=$ yes & 0.57 \\
\hline Age $75-79$ years & $0=$ no, $1=$ yes & 0.82 \\
\hline Age $80+$ years & $0=$ no, $1=$ yes & 1.01 \\
\hline Male & $0=$ no, $1=$ yes & 0.24 \\
\hline Inverse of BMl & 10/BMl & -1.60 \\
\hline Cancer, haematological & $0=$ no, $1=$ yes & 1.00 \\
\hline Cancer, non-haematological & $0=$ no, $1=$ yes & 0.26 \\
\hline Cerebrovascular disease & $0=$ no, $1=$ yes & 0.40 \\
\hline Diabetes & $0=$ no, $1=$ yes & 0.43 \\
\hline Hypertension & $0=$ no, $1=$ yes & 0.29 \\
\hline Kidney disease & $0=$ no, $1=$ yes & 0.69 \\
\hline Respiratory disease (excluding asthma) & $0=$ no, $1=$ yes & 1.17 \\
\hline rs112317747 & $0=\mathrm{T} / \mathrm{T}, 1=\mathrm{C} / \mathrm{T}, 2=\mathrm{C} / \mathrm{C}$ & 0.27 \\
\hline rs2034831 & $0=\mathrm{A} / \mathrm{A}, 1=\mathrm{C} / \mathrm{A}, 2=\mathrm{C} / \mathrm{C}$ & 0.24 \\
\hline rs112641600 & $0=\mathrm{C} / \mathrm{C}, 1=\mathrm{T} / \mathrm{C}, 2=\mathrm{T} / \mathrm{T}$ & -0.24 \\
\hline rs10755709 & $0=A / A, 1=G / A, 2=G / G$ & 0.12 \\
\hline rs118072448 & $0=\mathrm{T} / \mathrm{T}, 1=\mathrm{C} / \mathrm{T}, 2=\mathrm{C} / \mathrm{C}$ & -0.20 \\
\hline rs7027911 & $0=\mathrm{G} / \mathrm{G}, 1=\mathrm{A} / \mathrm{G}, 2=\mathrm{A} / \mathrm{A}$ & 0.10 \\
\hline rs71481792 & $0=\mathrm{A} / \mathrm{A}, 1=\mathrm{T} / \mathrm{A}, 2=\mathrm{T} / \mathrm{T}$ & -0.11 \\
\hline
\end{tabular}

306 Note: Body mass index (BMI) is calculated as $\mathrm{kg} / \mathrm{m}^{2}$; the inverse of BMl is calculated as 10 divided by

307 BMI. In the current analysis, we used rs 10905502 as a proxy for rs 71481792 and rs78654835 as a

308 proxy for rs 112317747. 
A

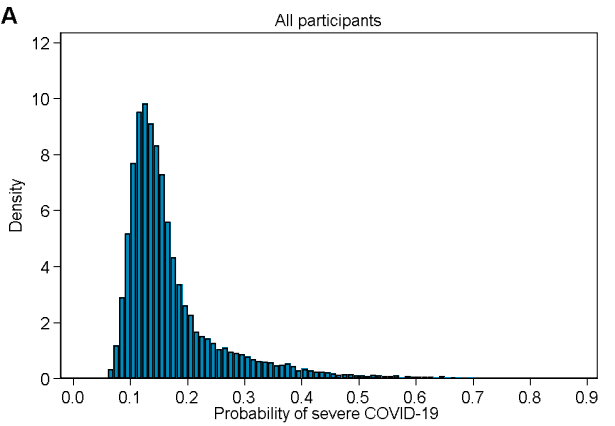

C

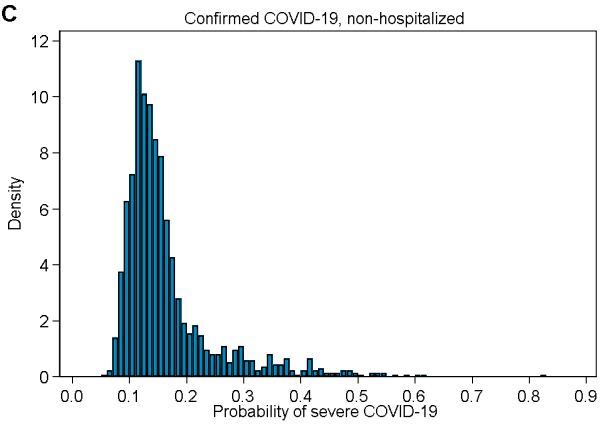

B

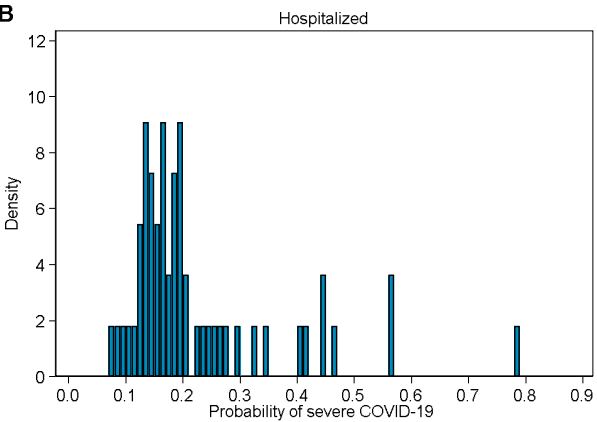

D

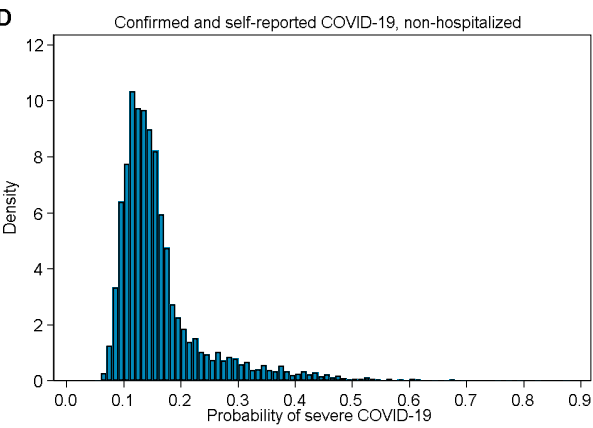

310 Supplementary Figure S1. Distribution of probability of severe COVID-19 in (A) all 311 Lifelines COVID-19 cohort participants, (B) hospitalized (cases), (C) non-hospitalized 312 confirmed COVID-19 (controls) and (D) non-hospitalized confirmed and self-reported 313 COVID-19 (controls).
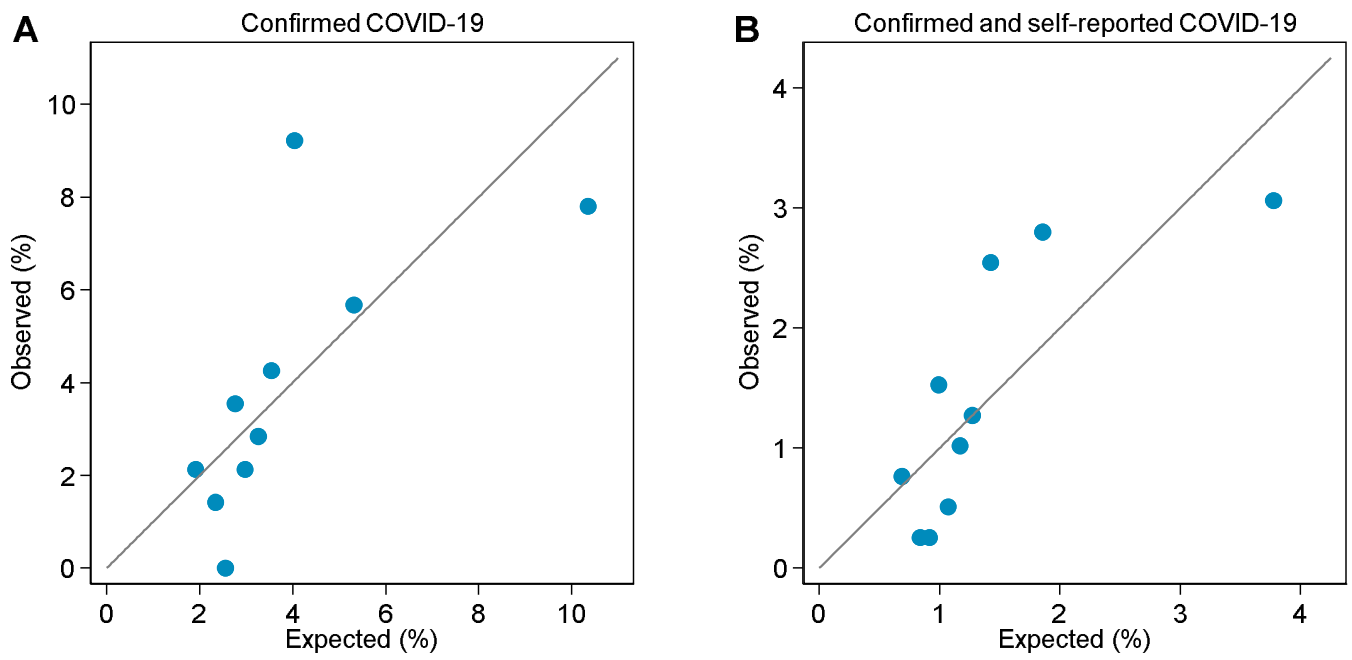

316 Supplementary Figure S2. Calibration plots for (A) the confirmed COVID-19 group and 317 (B) the confirmed and self-reported COVID-19 group. 